\title{
The Role of Lipids in Inflammation: Review of the Evolving Pathogenesis of Sickle Cell Disease
}

\author{
Anazoeze Jude Madu ${ }^{1^{*}}$, Nada Abuknesha ${ }^{2}$ and Kebreab Ghebremeskel ${ }^{2}$ \\ ${ }^{1}$ Department of Haematology and Immunology, College of Medicine, University of Nigeria Enugu campus, Nigeria \\ ${ }^{2}$ Lipidomics and Nutrition Research Center, London Metropolitan University, London, UK
}

*Corresponding author: Anazoeze J. Madu, Professor, Department of Haematology and Immunology, University of Nigeria Enugu Campus (UNEC), PMB 01129, Post code 400001, Enugu, Nigeria, Tel: +2348037861784; E-mail: anazoeze.madu@unn.edu.ng

Received date: June 30, 2015, Accepted date: July 30, 2015, Published date: August 6, 2015

Copyright: $@ 2015$ Madu AJ. This is an open-access article distributed under the terms of the Creative Commons Attribution License, which permits unrestricted use, distribution, and reproduction in any medium, provided the original author and source are credited.

\begin{abstract}
The pathologic features of sickle cell disease had been known in the past to be as a result of red cell abnormality leading to vascular occlusion, haemolysis and consequent anaemia. Recent knowledge has revealed numerous pathogenetic pathways involving leukocytes, platelets and the vascular endothelium. Complex interactions between the inflammatory cytokines and the membrane lipids in sickle cell present several pathogenetic processes affecting disease severity. The mechanisms of membrane fluidity, aggregation, adhesion and inflammation are strongly associated with membrane lipid constitution. The omega -3 fatty acids via incorporation into the lipid membrane have been found to play a central role in suppressing inflammation in several disease processes. Variations in disease severity have been shown to correspond with levels of fatty acid desaturases involved in the synthesis of these fatty acids. The genes coding for these substances can also be manipulated to achieve a favorable outcome and may provide several possible therapeutic and prophylactic access points This review aims at exploring these delicate interactions and proffering possible targets to ameliorate disease features. The information and referenced publications quoted in this review were obtained from the PubMed Central database, using the search keywords; inflammation, sickle cell, fatty acids and cytokines.
\end{abstract}

Keywords: Membrane lipids; Sickle cell disease; Inflammation; Omega-3 fatty acids; Cytokines; Fatty acid desaturases

\section{Introduction}

Sickle cell disease is an inherited disorder in which the mutant globin gene produces a less efficient haemoglobin molecule, causing depreciation in the resilience and flexibility of the red cells. Recent advances have unearthed several other effects of this supposed mutant globin gene. These effects are now known to include increased expression and binding of the cellular adhesion molecules to ligands on the vascular endothelium [1] a process similar to what is observed during inflammation [2]. This process involves the neutrophils, monocytes, lymphocytes and platelets as well as the reticulocytes and red cells. The background inflammatory process tends to be exacerbated during episodes of vaso-occlusion and has been proposed to actually initiate them. Inflammation is regarded as the response of living tissue to any form of assault. This response has both vascular and cellular components, with a myriad of chemical interactions mediating the process.

All cells are bound by a membrane considered to be the 'barrier of life', as it is a structure that isolates living cells from their surroundings [3]. One of the main components of all cellular membranes in all living cells, are phospholipids, which make up over $40 \%$ of the cell membrane bi-layer. This phospholipid bi-layer, with its interspersed transmembrane proteins acts as receptors of ligands. The asymmetrical distribution of membrane lipids in this phospholipid layer is important in the integrity and survival of most cells. In sickle cell anaemia, there exists an alteration in the distribution of membrane lipids, with exposure of phosphatidyl serine (PS) in some red cells in circulation [4]. This distorts the membrane and consequently exposes and activates cell adhesion receptors as well as the binding sites of certain enzymes like promthrombinase [5]. It also initiates apoptosis of red cells and its removal by the splenic macrophages. The concentration of the membrane lipids differs across various locations on the membrane, with some regions showing increased concentration of certain fatty acids. These areas are known as "rafts" or "microdomains".

The Omega-3 fatty acids, particularly eicosapentaenoic acid (EPA) and docosapentaenoic acid (DHA), have been observed to have antiinflammatory effects. Dietary omega-3 fatty acids are easily incorporated to a large extent into the cell membrane lipids and thus influence the composition of the rafts. The mechanisms by which these poly-unsaturated fatty acids carry out these functions are not completely understood. However, recent studies have shown that intake of $\omega-3$ fatty acids decreases levels of $C$ reactive proteins (CRP), IL-6 and tumor necrosis factors (TNF- $\alpha$ ) [6]. These actions are hypothesized to be effected mainly via regulations of the transcription factors; nuclear factor $\kappa$ and peroxisome proliferator activated receptor. Other inflammatory markers which are reduced such as; monocytes chemo-attractant protein 1 (MCP-1), intercellular adhesion molecule 1 (ICAM-1) and lipoprotein-associated phospholipase A2 (LpPLA-2), also play important roles in increasing oxidative stress [7].

The rate limiting enzymes in the production of these fatty acids are the $\Delta-3$ and $\Delta-6$ fatty acid desaturases. The depreciation in activity of these enzymes may be responsible for the depletion of the PUFAs in sickle cell disease (SCD) patients, which occurs to a greater extent in those with severe disease. Protein synthesis is consequent upon DNA transcription and any mutation in genes coding for these enzymes will 
invariably affect these function. This review aims to explore the inflammatory pathways involved in SCD as well as the impact of the PUFAs, desaturases and their complex interactions with regards to the myriad of phenotypic presentation observed. The review also focuses on the background genetic variations with regards to FADs activity that may be responsible for these observed phenotypic differences as well as suggest possible therapeutic implications.

\section{Inflammatory proteins and the role of membrane lipids}

The red cell membrane is made of a phospholipid bi-layer and the underlying structural protein scaffolding lattice arranged in the format regarded as the vertical and horizontal associations. The membrane phospholipids are made up of a polar head and a non-polar fatty acid tail. There are proteins embedded in this bi-layer that tether them to the underlying protein lattice. The lipids move rapidly within this bilayer and the areas where a high concentration of these lipids and proteins occur are called 'micro-domains' or rafts. [8]. The lipid contents of these micro-domains to some extent affect the function of the membrane proteins/ receptors embedded in them $[9,10]$. These rafts which take part in signal transduction are rich in sphingomyelin, cholesterol and saturated glycerol phospholipids [10]. Certain dietary lipids are easily incorporated into the membrane and reduction in membrane cholesterol content tends to cause dissolution of these rafts $[11,12]$.

There also exists an active exchange of lipids between the inner and outer leaves of the bi-layer, this is not random and is controlled by certain proteins in the membrane. Sphingomyelin (SM) and phosphatidyl choline (PC) - the choline containing phospholipids, are more abundant on the outer layer, while phosphatidyl ethanolamine (PE) and phosphatidyl serine (PS) predominate on the inner layer. This balance is maintained by the ATP- dependent membrane transporter called 'flippase', which is a $\mathrm{Mg}^{2+}$-ATPase bound to the membrane [13]. Recently the enzyme amino-phospholipase translocase has also been discovered to be important in the maintenance of the lipid distribution [14]. This dynamic balance is necessary for the optimal functioning and survival of most eukaryotic cells.

The phospholipids in the cellular membrane contain fatty acids (FA), and the type of diet an individual consume will directly determine the type of FA (saturated or un-saturated) that will be incorporated into the phospholipid bi-layer, and consequently will define the 'structural integrity' and physical characteristics of the cellular membrane $[3,15,16]$. The lipid composition of the cellular membrane will therefore, govern the effectiveness of its fluidity and hence its membrane fluidity index (MFI) [15-17]. The PUFA's (EPA and DHA) have been exhibited in many studies to increase the MFI and thus create a peculiar environment for the membrane proteinstransporters, ion channels and receptors and by doing this influence their activity. The physical state of the membrane needs to be optimally balanced in terms of fluidity [16] in order for cells to carry out their regular functions and activities maximally. In addition, ideal MF will decrease the cells susceptibility to damage and death significantly[3,15]. These areas of the lipid membrane bi-layer, rich in cholesterol, sphingomyelin and saturated glycerol phospholipids are called rafts or micro-domains. Apart from their membrane function these rafts are also known to influence intra-cellular signaling pathways, $[8,18]$ thus affecting gene expression via their action on transcription factors $[19,20]$. These fatty acids are known to effect these actions by acting on transcription factors; nuclear factor $\kappa$, binding protein for the sterol regulatory element [21] and peroxisome proliferator activator receptor (PPAR) $\alpha$ and $\gamma[22,23]$. This ability has been ascribed to DHA and EPA to varying degrees depending on the nature of the cell. Any reduction in the incorporation of cholesterol into the membrane is known to cause dissolution of these rafts.

Nuclear factor- $\kappa \mathrm{B}$ upon activation is a potent transcription factor for several inflammatory cytokines; cycloxygenase (COX)-2, adhesion molecules and nitric oxide synthase. Upon activation by bacterial endotoxins, oxidative stress, UV light or inflammatory cytokines, it moves from the cytosol to the nucleus to enhance production of proinflammatory cytokines. Several studies have shown that EPA and DHA inhibit this inflammatory action in monocytes [24], dendritic cells [19], macrophages [25] and endothelial cells [26]. They inhibit monocyte NF- $\kappa B$ and consequently modulate transcription of TNF- $\alpha$, [22] (which plays a central role in inflammation) this influences the other factors to indirectly control fatty and triacyl glycerol metabolism. The PPARs are transcription factors which regulate gene expression by binding to retinoic-X-receptors, which are bound by the ligand; 6 cisretinoic acid [27]. The genes coding for several enzymes involved in $\beta$ oxidation are regulated by the PPAR- $\alpha$ isoform, predominantly found in the liver. PPAR- $\gamma$ is found in inflammatory cells and adipocytes and is involved in increasing insulin sensitivity and regulating production of inflammatory cytokines [28]. PPARs are activated by PUFAs, especially DHA to cause decreased production of IL- 6 and TNF- $\alpha$ as well as effects its metabolic role on adipocytes [29]. This is proposed to be the mechanism underlying the effect of omega-3 PUFAs in reducing inflammation and plasma glycerol and improving insulin sensitivity in diabetics.

Other effects of the variations in the lipid membrane are mediated via the availability of arachidonic acid, an important substrate in the production of very important bio-active lipid end products prostaglandins, thromboxanes and leukotrienes. These eicosanoids are known mediators of inflammation causing, platelet aggregation, smooth muscle contraction and vaso-dilatation. Intake of long chain fatty acids (DHA and EPA) have been observed to cause decrease in the production of these pro-inflammatory eicosanoids from arachidonic acid as well as production of weaker or less inflammatory analogues - prostaglandin E3 and thromboxanes A3 [30]. Recent studies have shown that EPA and DHA are also substrates in complex biosynthetic pathways that give rise to anti-inflammatory mediators known as resolvins, protectins/neuroprotectins and maresins [31-33]. These mediators also take part in immuno-modulation and are currently being hypothesized to be the major pathway through which polyunsaturated fatty acids carry out their anti-inflammatory actions.

The omega 3 fatty acids bind to G-protein coupled receptors; GPR40 and GPR120 are found on Adipocytes and inflammatory cells [34]. The GPR 120 acts via its agonist, GW9508 to inhibit response of macrophages to endotoxins [35]. This is achieved by activating the inhibitory subunit $(\mathrm{I} \kappa \mathrm{B})$ of NF- $\kappa \mathrm{B}$, and thereby inhibiting production of TNF- $\alpha$ and IL-6. DHA and EPA are known to activate the genes for GPR120, and their anti-inflammatory effects are to some extent dependent on this pathway. Previous studies have shown that the antiinflammatory effects of DHA, was absent in GPR120 knockdown cells, [27] further substantiating this assertion. This implies that the inhibitory effects of DHA on NF- $\kappa B$, most likely occurred via the GPR120 pathway. However, both pathways may be involved in varying degrees in different types of cells. 


\section{Alteration in Membrane Lipids in Sickle Cell Disease}

In mammalian cells this peculiar distribution of the membrane lipids is of utmost importance in the maintenance of its integrity. The red cells in their journey through areas of high oxygen tension are particularly susceptible to the development of reactive oxygen species [36,37]. Membrane damage due to increased oxidant stress as a result of formation of oxygen free radicals and outstripping of the antioxidant mechanisms is the main mechanisms of membrane damage in SC. Intravascular haemolysis of sickle red cells also release haeme protein and the enzyme arginase which mops up nitrous oxide and arginine, respectively. This produces further oxidative stress, resulting in production of more oxygen free radicals. These reactive oxygen species disrupt the apolar acyl chains of the membrane phospholipids and thereby making close-packing of the lipids impossible [29]. These oxidized portions are usually removed by the phospholipases via a deacylation process and replaced by selective uptake from plasma lipids in an ATP-dependent process called re-acylation. Inability to initiate this repair process leads to excessive exposure of PS on the outer leaf of the membrane. This exposure in platelets has been noted to be the initial step in activation of the coagulation cascade [38] and its occurrence in red cells lead to removal by macrophages as well as interactions with other cells and vascular endothelium [5]. In haemoglobinopathies the altered haemoglobin produces added oxidant stress on the anti-oxidant system, as well as the repair process of the membrane phospholipid layer[39,40].

Homozygous S individuals have been shown to have a reduced capacity to handle oxidative stress. This is coupled with their enhanced propensity of the red cell environment to generate oxygen free radical due to its iron content and passage through regions of high oxygen tension, as it traverses the vasculature. There is also an observed reduction in the activity of plasma as well as membrane bound antioxidants notably, plasma retinol, $\alpha$-tocopherol and $\beta$-carotene and the red cell $\mathrm{Cu} / \mathrm{Zn}$-superoxide dismutase and Se-glutathione peroxidase [41]. The changes in these anti-oxidants have also been observed to correlate with the concentration of the fatty acids DHA and EPA [36], it has not yet become clear which is the cause or effect of the other. However, it has been proposed that the increased generation of oxygen free radicals leads to peroxidation of the more susceptible lipids- $n-5$ and $n-6$ fatty acids, and their subsequent depletion. It will however need to be demonstrated that an increase in the antioxidant levels will lead to a subsequent increase in omega 3 fatty acid levels.

\section{Background Inflammation in Sickle Cell}

Sickle cell patients with a high leukocyte counts have higher predisposition to develop vaso-occlusive crises as well as several other chronic complications of sickle cell disease. High levels of proinflammatory cytokines have been observed in these patients even while in steady state [42-44]. There's increasing evidence of the overexpression of the platelet selectin and its ligand (PSGL-1 or CD162), leukocyte selectin (CD62), $\beta 2$ integrin and platelet endothelial cell adhesion molecule-1[6]. The vascular endothelium is also activated with increased expression of inter-cellular adhesion molecule-1 (ICAM-1) and vascular cell adhesion molecule-1 (VCAM-1). This background state of smoldering inflammation persists, though on a lower scale in sickle cell patients in steady state. The initial contact and rolling of the leukocytes on the endothelium is mediated via CD62L, while the firmer attachment and diapedesis are mediated by $\beta$ integrins and CD31 (PECAM-1 platelet endothelial cell adhesion molecule). These leukocytes then bind to each other and to the platelets, red cells and reticulocytes to form larger aggregates, capable of occluding vascular lumen. This is similar to the initial steps observed in acute inflammatory processes and also involves endothelial activation via TNF- $\alpha$ and interleukin- $1 \beta$, released by monocytes. Periodic exacerbation of these events occur as vasoocclusive crisis when external stimuli, like infections or physiologic stress, cause greater amounts inflammatory cytokines to be released by activated leukocytes.

These cellular adhesion molecules and their ligands occur as transmembrane proteins and receptors, and their concentrations as well as activity are most likely affected by the lipid composition of the membrane. A large community-based study has shown an inverse relationship between the level of inflammatory biomarkers (notably IL-6, TNF receptor, ICAM-1 and P-selectin) and the blood EPA and DHA levels $[45,46]$. Drugs like hydroxyurea (HU) have been found to cause improvement even in patients in whom there was no significant change in their haemoglobin F levels. Apart from reducing the neutrophil count (and thereby depleting the overall number of inflammatory cells and ligands available), it has been found to also reduce expression of adhesion molecules by reticulocytes [47] and inhibit the translocation of PS to the outer membrane leaf of the red cell [48].

Possible genetic variations underlying observed changes in lipid metabolism and their possible influence on disease phenotype.

There are peculiar differences which exist in the genome of minority of individuals; these variations may lead to alterations in quantity or activity of certain enzymes. These small scale genetic variations include; single, double or multi-nucleotide polymorphisms (SNP), as well as microindels. Microindels are additions or deletions of extra nucleotides as well as occurrence of repetitions of 1 to 50 nucleotides, or combinations of both within the sequence. SNPs are single base changes in the DNA sequence, seen in less than $1 \%$ of individuals, which occur at an approximate frequency of 1 in $1000 \mathrm{~kb}$ [49] and may or may not be of clinical significance. These mutations may occur in the coding region of genes encoding the fatty acid desaturases or inflammatory cytokines and thereby confer some phenotypic differences in terms of disease severity. Previous studies have observed that SNPs and microindels have been implicated in drug metabolism via their encoding gene- CYP2D6 with loss of enzyme activity and slow metabolism [50,51]. Alterations in the FADS enzyme genes are associated with the variations in the activity of these enzymes [52,53] as well as the K-ras gene in some other patient groups. Polymorphism in the genes coding for the FADS enzymes have been discovered in genome wide association studies by Guan et al. [54].

The FADs enzymes as well as phospholipase-A 2, play a pivotal role in the production of the anti-inflammatory omega- 3 fatty acids and pro-inflammatory omega- 6 fatty acids. Previous studies have shown low activity of the FADS enzymes as well as omega- 3 fatty acids in sickle cell patients with severe disease. Meta-analysis of genes coding for the Omega- 6 fatty acids have also revealed that gene loci in other chromosomes as well as distant SNPs may also affect the level and activity of this enzyme [54]. Modifications and changes in the genes coding for these enzymes will lead to loss of FADS enzyme activity or quantity and invariably lead to increased production of IL- 6 and TNF$a$, with subsequent worsening of the clinical features of the disease. We hypothesize this as a possible explanation for the variations in the phenotypic manifestations observed in sickle cell disease. 


\section{Future Therapeutic Implications}

Therapeutic targets have so far concentrated on the red cell-induced vaso-occlusion and the haemolysis associated with the disease. This has included attempts at improving hydration, ameliorating antioxidant effects, increasing synthesis of $\mathrm{HbF}$, replenishing plasma arginine and improving red cell rheology. This section will however focus on the possible prospective anti-inflammatory and fatty acid metabolic options.

\section{Omega-3 fatty acids}

The omega- 3 fatty acids play a pivotal role in the synthesis of less inflammatory end products of the cycloxygenase pathway, but more importantly suppress transcriptions of the genes of potent cytokines, which mediate inflammation. This has been shown by several studies in past in different patient groups, including SC [6] and diabetes, [55] where they have been noted to induce amelioration of the symptoms and improve well-being [56]. Dietary DHA and EPA, after incorporation into the cell membrane, have also been shown in previous studies to increase membrane fluidity and thus affect the activity of the transmembrane proteins [12]. These are natural products with little or no side effects, which given at certain doses, positively influence metabolic pathways to favor reduced inflammation, and consequently reduce disease severity. It must always be remembered that inflammation is an innate defensive mechanism and that it's prolonged and sustained suppression, may likely lead to some untoward effects. However the PUFAs as opposed to the saturated FAs competitively bind to the Toll-like receptor 4, through which it influences the transcription of NF- $\mathrm{k}$, and consequently the COX-2 pathway, while the COX-1 pathway remains relatively untouched [25]. In this way the most beneficial inflammatory proteins and cytokines are to a large extent preserved. The therapeutic threshold for each disease entity, as well as the 'ceiling dose', beyond which severe adverse effects occur, has not yet been adequately established. The ratio of omega- 3 to omega- 6 fatty acids has also been observed to be of importance, since the excess of omega-6 FAs favors the increased inflammation, insulin resistance and macrophage infiltration[57]. Future research will most likely focus on the synthesizing omega-3 fatty acid analogues and mixtures of various PUFAs in order to achieve an effective dosing and metabolic equilibrium.

\section{Fatty Acid Desaturases and their encoding genes}

These enzymes occur as the rate-limiting enzymes in the production of the omega- 3 fatty acids [58]. Increase in the production of these fatty acids can also be achieved by increasing the FADS enzyme activity or alternatively targeting their coding genes or transcription factors [59]. The FADS1 and 2 enzymes are encoded by exon 13 on chromosome 11q12.2-13.1for FADS1 and exon 14 on chromosome 11q12.2, for FADS 2. Alterations in the form of polymorphisms affecting these genes will affect their transcription $[52,53]$ and may be utilized to obtain an increase in production of PUFAs. Also these areas may be targeted by plasmids containing specific desired sequences which are capable of converting severe to mild disease phenotypes.

\section{Hydroxyurea and its analogues}

Majority of the effects of HU on sickle cell disease is carried out via its ability to reduce neutrophil count, increase HbF levels [60] and reduce expression of cellular adhesion molecules[48,61]. Hydroxycarbamide also reduces the exposure of phosphatidyl serine on the surface of platelets and red cells [48]. It has been observed that $\mathrm{HU}$ does not cause an increase in $\mathrm{HbF}$ in all SCA patients, [62] and does not completely protect against end organ damage [63]. Previous research has also shown that HU mobilizes arachidonic acid from the inner leaflet of the red cell membrane via its action on COX-2 and cytoplasmic PLA-2 [64]. The eicosanoids DHA which is generated as a result of this process is thought to play a part in the therapeutic improvement seen in patients on HU. However the observation of a reduction in fertility and alterations in sex hormones after 6 months of therapy raises some concerns [65]. There arises the need to increase efforts to produce a safer analogue of this very important molecule, which may also be used in combination with drugs acting via other pathways at less toxic doses.

\section{Inhibition of adhesion molecules}

Several adhesion molecules are being targeted in on-going trials. The selectin inhibitors, Rivipansel and low molecular weight heparin both act by inhibiting platelet, leukocyte and endothelial adhesion[46]. $\mathrm{HU}$ to a large extent also diminishes the cellular and endothelial adhesion molecules by reducing the total number of white cells available for adhesion [66]. Omega 3 fatty acids on the other hand suppress the expression of the adhesion molecules $\beta 2$ - integrin (CD-11b) and L-selectin (CD62L) in monocytes and granulocytes [6]. Intravenous gamma globulins as well as $\beta 3$-integrin inhibitors are also being tested [63]. These molecules are quite appropriate for both prevention and treatment of end organ damage, in some cases. However the search for a treatment of the most prevalent form of crisis- the acute vaso-occlusive (bone pain) still remains elusive. Emergency therapeutic drugs are still required which should be capable of adequately aborting acute vaso-occlusion.

\section{Resolvins/Protectins pathway activators}

The resolvins, protectins and maresins are end product of the metabolism of DHA and to a lesser extent EPA, via complex biosynthetic pathways. They seem to play an important role in suppressing inflammation and have been hypothesized to be the major pathway through which n-3 fatty acids achieve these ends [67]. They also play a role in modulating immune functions via their direct effect on gene expression, targeting genes coding for cytokines, cyclooxygenase, nitric oxide synthetase and metalloproteinases [20,68]. Therefore these mediators or their analogues can be given directly, as a form of more specific therapeutic targeting, to achieve these desirable ends.

\section{Targeting genes for transcription factors in the inflammatory pathway}

Down-regulation of the transcription factor NF- $\mathrm{kB}$ has been noted repeatedly to be responsible for majority of the actions of most important prospective molecules in trial. Synthetic products targeting this transcription factor can be developed and tried in the clinical setting. Gene therapy has been on the lime-light for some years now, but newer target genes should be explored. Ex-vivo manipulation of autologous haemopoietic stem cells have also been tried, especially for patients who do not have a matched donor [69]. Newer and safer viral vectors have also been developed to overcome the occurrence of genotoxicity. The beta globin gene chain in erythropoietic stem cells had been used in the past, but current and future researches are 
focusing on the pluripotent stem cells [70]. It may also be helpful target the inflammatory genes if these makes transfection and uptake more tolerable, as this may serve to ameliorate disease severity.

\section{Declaration of Interests}

The authors report no declaration of interests.

\section{References:}

1. Okpala I (2006) Leukocyte adhesion and the pathophysiology of sickle cell disease. Curr Opin Hematol 13: 40-44.

2. Niu X, Nouraie M, Campbell A, Rana S, Minniti CP, et al. (2009) Angiogenic and inflammatory markers of cardiopulmonary changes in children and adolescents with sickle cell disease. PLoS One 4: e7956.

3. Ibarguren M, López DJ, Escribá PV (2014) The effect of natural and synthetic fatty acids on membrane structure, microdomain organization, cellular functions and human health. Biochim Biophys Acta 1838: 1518-1528.

4. Kuypers FA (2007) Membrane lipid alterations in hemoglobinopathies. Hematology Am Soc Hematol Educ Program .

5. Zwaal RF, Schroit AJ (1997) Pathophysiologic implications of membrane phospholipid asymmetry in blood cells. Blood 89: 1121-1132.

6. Daak AA, Elderdery AY, Elbashir LM, Mariniello K, Mills J, et al. (2015) Omega 3 (n-3) fatty acids down-regulate nuclear factor-kappa B (NF-Î०B) gene and blood cell adhesion molecule expression in patients with homozygous sickle cell disease. Blood Cells Mol Dis 55: 48-55.

7. Calder PC (2015) Marine omega-3 fatty acids and inflammatory processes: Effects, mechanisms and clinical relevance. Biochim Biophys Acta 1851: 469-484

8. Simons K, Gerl MJ (2010) Revitalizing membrane rafts: new tools and insights. Nat Rev Mol Cell Biol 11: 688-699.

9. Ventimiglia LN, Alonso MA (2013) The role of membrane rafts in Lck transport, regulation and signalling in T-cells. Biochem J 454: 169-179.

10. Simmons C, Ingham V, Williams A, Bate C (2014) Platelet-activating factor antagonists enhance intracellular degradation of amyloid-beta42 in neurons via regulation of cholesterol ester hydrolases. Alzheimers Res Ther 6:15.

11. Torres M, Price SL, Fiol-Deroque MA, Marcilla-Etxenike A, Ahyayauch $\mathrm{H}$, et al. (2014) Membrane lipid modifications and therapeutic effects mediated by hydroxydocosahexaenoic acid on Alzheimer's disease. Biochim Biophys Acta 1838: 1680-1692.

12. Calder PC, Yaqoob P, Harvey DJ, Watts A, Newsholme EA (1994) Incorporation of fatty acids by concanavalin A-stimulated lymphocytes and the effect on fatty acid composition and membrane fluidity. Biochem J 300 : 509-518.

13. Soupene E, Kuypers FA (2006) Identification of an erythroid ATPdependent aminophospholipid transporter. Br J Haematol 133: 436-438.

14. Sarkar A, Sengupta D, Mandal S, Sen G, Dutta Chowdhury K, et al. (2015) Treatment with garlic restores membrane thiol content and ameliorates lead induced early death of erythrocytes in mice. Environ Toxicol 30: 396-410.

15. Yehuda S, Rabinovitz S, Carasso RL, Mostofsky DI (2002) The role of polyunsaturated fatty acids in restoring the aging neuronal membrane. Neurobiol Aging 23: 843-853.

16. Yehuda S, Rabinovitz S, Mostofsky DI (1999) Essential fatty acids are mediators of brain biochemistry and cognitive functions. J Neurosci Res 56: $565-570$

17. Dyall SC, Michael-Titus AT (2008) Neurological benefits of omega-3 fatty acids. Neuromolecular Med 10: 219-235.

18. Pike LJ (2003) Lipid rafts: bringing order to chaos. J Lipid Res 44: 655-667.

19. Draper E, Reynolds CM, Canavan M, Mills KH, Loscher CE, et al. (2011) Omega-3 fatty acids attenuate dendritic cell function via NF-B independent of PPAR $\gamma$. J Nutr Biochem 22: 784-790.
20. Calder PC (2013) Long chain fatty acids and gene expression in inflammation and immunity. Curr Opin Clin Nutr Metab Care 16: $425-433$.

21. Jump DB (2008) N-3 polyunsaturated fatty acid regulation of hepatic gene transcription. Curr Opin Lipidol 19: 242-247.

22. Novak TE, Babcock TA, Jho DH, Helton WS, Espat NJ (2003) NF-kappa B inhibition by omega -3 fatty acids modulates LPS-stimulated macrophage TNF-alpha transcription. Am J Physiol Lung Cell Mol Physiol 284: L84-89.

23. Göttlicher M, Widmark E, Li Q, Gustafsson JA (1992) Fatty acids activate a chimera of the clofibric acid-activated receptor and the glucocorticoid receptor. Proc Natl Acad Sci U S A 89: 4653-4657.

24. Lo CJ, Chiu KC, Fu M, Lo R, Helton S (1999) Fish oil decreases macrophage tumor necrosis factor gene transcription by altering the NF kappa B activity. J Surg Res 82: 216-221.

25. Lee JY, Sohn KH, Rhee SH, Hwang D (2001) Saturated fatty acids, but not unsaturated fatty acids, induce the expression of cyclooxygenase-2 mediated through Toll-like receptor 4. J Biol Chem 276: 16683-16689.

26. Khalfoun B, Thibault F, Watier H, Bardos P, Lebranchu Y (1997) Docosahexaenoic and eicosapentaenoic acids inhibit in vitro human endothelial cell production of interleukin-6. Adv Exp Med Biol 400B: 589-597.

27. Hasan AU, Ohmori K, Konishi K, Igarashi J, Hashimoto T, et al. (2015) Eicosapentaenoic acid upregulates VEGF-A through both GPR120 and PPAR $\gamma$ mediated pathways in 3T3-L1 adipocytes. Mol Cell Endocrinol 406: $10-18$.

28. Szanto A, Nagy L (2008) The many faces of PPARgamma: antiinflammatory by any means? Immunobiology 213: 789-803.

29. Kong W, Yen JH, Vassiliou E, Adhikary S, Toscano MG, et al. (2010) Docosahexaenoic acid prevents dendritic cell maturation and in vitro and in vivo expression of the IL-12 cytokine family. Lipids Health Dis 9: 12.

30. Moncada S, Vane JR (1979) The role of prostacyclin in vascular tissue. Fed Proc 38: 66-71.

31. Bannenberg G, Serhan CN (2010) Specialized pro-resolving lipid mediators in the inflammatory response: An update. Biochim Biophys Acta 1801: 1260-1273.

32. Serhan CN, Chiang N, Van Dyke TE (2008) Resolving inflammation: dual anti-inflammatory and pro-resolution lipid mediators. Nat Rev Immunol 8: 349-361.

33. Serhan CN, Yacoubian S, Yang R (2008) Anti-inflammatory and proresolving lipid mediators. Annu Rev Pathol 3: 279-312.

34. Liu Y, Chen LY, Sokolowska M, Eberlein M, Alsaaty S, et al. (2014) The fish oil ingredient, docosahexaenoic acid, activates cytosolic phospholipase $A_{2}$, via GPR120 receptor to produce prostaglandin $E_{2}$, and plays an anti-inflammatory role in macrophages. Immunology 143: 81-95.

35. Oh DY, Talukdar S, Bae EJ, Imamura T, Morinaga H, et al. (2010) GPR120 is an omega-3 fatty acid receptor mediating potent antiinflammatory and insulin-sensitizing effects. Cell 142: 687-698.

36. Ren H, Ghebremeskel K, Okpala I, Lee A, Ibegbulam O, et al. (2008) Patients with sickle cell disease have reduced blood antioxidant protection. International journal for vitamin and nutrition research Internationale Zeitschrift fur Vitamin- und Ernahrungsforschung Journal international de vitaminologie et de nutrition. 78:139-147.

37. Kehrer JP (2000) The Haber-Weiss reaction and mechanisms of toxicity. Toxicology 149: 43-50.

38. Nur E, Brandjes DP, Teerlink T, Otten HM, Oude Elferink RP, et al. (2012) $\mathrm{N}$-acetylcysteine reduces oxidative stress in sickle cell patients. Ann Hematol 91: 1097-1105.

39. Hebbel RP (1984) Erythrocyte autoxidation and the membrane abnormalities of sickle red cells. Prog Clin Biol Res 159: 219-225.

40. Schrier SL, Centis F, Verneris M, Ma L, Angelucci E (2003) The role of oxidant injury in the pathophysiology of human thalassemias. Redox Rep 8: 241-245. 
41. Schacter LP, DelVillano BC, Gordon EM, Klein BL (1985) Red cell superoxide dismutase and sickle cell anemia symptom severity. Am J Hematol 19: 137-144.

42. Pathare A, Al Kindi S, Alnaqdy AA, Daar S, Knox-Macaulay H, et al. (2004) Cytokine profile of sickle cell disease in Oman. Am J Hematol 77: 323-328.

43. Croizat H (1994) Circulating cytokines in sickle cell patients during steady state. Br J Haematol 87: 592-597.

44. Bourantas KL, Dalekos GN, Makis A, Chaidos A, Tsiara S, et al. (1998) Acute phase proteins and interleukins in steady state sickle cell disease. Eur J Haematol 61: 49-54.

45. Fontes JD, Rahman F, Lacey S, Larson MG, Vasan RS, et al. (2015) Red blood cell fatty acids and biomarkers of inflammation: a cross-sectional study in a community-based cohort. Atherosclerosis 240: 431-436.

46. Okpala I (2015) Investigational selectin-targeted therapy of sickle cell disease. Expert Opin Investig Drugs 24: 229-238.

47. Styles LA, Lubin B, Vichinsky E, Lawrence S, Hua M, et al. (1997) Decrease of very late activation antigen- 4 and CD36 on reticulocytes in sickle cell patients treated with hydroxyurea. Blood 89: 2554-2559.

48. Covas DT, de Lucena Angulo I, Vianna Bonini Palma P, Zago MA (2004) Effects of hydroxyurea on the membrane of erythrocytes and platelets in sickle cell anemia. Haematologica 89: 273-280.

49. Levy S, Sutton G, Ng PC, Feuk L, Halpern AL, et al. (2007) The diploid genome sequence of an individual human. PLoS Biol 5: e254.

50. Eichelbaum M, Ingelman-Sundberg M, Evans WE (2006) Pharmacogenomics and individualized drug therapy. Annu Rev Med 57: 119-137.

51. Ingelman-Sundberg M, Sim SC (2010) Pharmacogenetic biomarkers as tools for improved drug therapy; emphasis on the cytochrome P450 system. Biochem Biophys Res Commun 396: 90-94.

52. Nwankwo JO, Spector AA, Domann FE (2003) A nucleotide insertion in the transcriptional regulatory region of FADS2 gives rise to human fatty acid delta-6-desaturase deficiency. J Lipid Res 44: 2311-2319.

53. Merino DM, Ma DW, Mutch DM (2010) Genetic variation in lipid desaturases and its impact on the development of human disease. Lipids Health Dis 9: 63.

54. Guan W, Steffen BT, Lemaitre RN, Wu JH, Tanaka T, et al. (2014) Genome-wide association study of plasma N6 polyunsaturated fatty acids within the cohorts for heart and aging research in genomic epidemiology consortium. Circ Cardiovasc Genet 7: 321-331.

55. Elwakeel NM, Hazaa HH (2015) Effect of omega 3 fatty acids plus lowdose aspirin on both clinical and biochemical profiles of patients with chronic periodontitis and type 2 diabetes: a randomized double blind placebo-controlled study. J Periodontal Res [Epub ahead of print].

56. Yessoufou A, Nekoua MP, Gbankoto A, Mashalla Y, Moutairou K (2015) Beneficial effects of omega-3 polyunsaturated Fatty acids in gestational diabetes: consequences in macrosomia and adulthood obesity. J Diabetes Res 731434.

57. Lopez-Vicario C, Gonzalez-Periz A, Rius B, Moran-Salvador E, GarciaAlonso V, et al. (2014) Molecular interplay between Delta5/Delta6 desaturases and long-chain fatty acids in the pathogenesis of nonalcoholic steatohepatitis. Gut 63:344-355.

58. Tosi F, Sartori F, Guarini P, Olivieri O, Martinelli N (2014) Delta-5 and delta-6 desaturases: crucial enzymes in polyunsaturated fatty acid-related pathways with pleiotropic influences in health and disease. Adv Exp Med Biol 824: 61-81.

59. Roke K, Ralston JC, Abdelmagid S, Nielsen DE, Badawi A, et al.(2013) Variation in the FADS1/2 gene cluster alters plasma n-6 PUFA and is weakly associated with hsCRP levels in healthy young adults. PLEFA 89: 257-263.

60. Calzolari R, Pecoraro A, Borruso V, Troia A, Acuto S, et al. (2008) Induction of gamma-globin gene transcription by hydroxycarbamide in primary erythroid cell cultures from Lepore patients. Br J Haematol 141: 720-727.

61. Odièvre MH, Bony V, Benkerrou M, Lapouméroulie C, Alberti C, et al. (2008) Modulation of erythroid adhesion receptor expression by hydroxyurea in children with sickle cell disease. Haematologica 93: 502-510.

62. McGann PT, Ware RE (2011) Hydroxyurea for sickle cell anemia: what have we learned and what questions still remain? Curr Opin Hematol 18: 158-165.

63. Conran N (2015) Prospects for early investigational therapies for sickle cell disease. Expert Opin Investig Drugs 24: 595-602.

64. Daak AA, Ghebremeskel K, Elbashir MI, Bakhita A, Hassan Z, et al. (2011) Hydroxyurea therapy mobilises arachidonic Acid from inner cell membrane aminophospholipids in patients with homozygous sickle cell disease. J Lipids 2011: 718014.

65. Berthaut I, Guignedoux G, Kirsch-Noir F, de Larouziere V, Ravel C, et al. (2008) Influence of sickle cell disease and treatment with hydroxyurea on sperm parameters and fertility of human males. Haematologica 93: 988-993.

66. Okpala I (2004) The intriguing contribution of white blood cells to sickle cell disease - a red cell disorder. Blood Rev 18: 65-73.

67. Calder PC (2013) n-3 fatty acids, inflammation and immunity: new mechanisms to explain old actions. Proc Nutr Soc 72: 326-336.

68. Dhingra AK, Chopra B, Dass R, Mittal SK (2015) An update on Antiinflammatory Compounds: A Review. Antiinflamm Antiallergy Agents Med Chem .

69. Ghosh S Thrasher AJ, Gaspar HB (2015) Gene therapy for monogenic disorders of the bone marrow. Br J Haematol .

70. Finotti A, Breda L, Lederer CW, Bianchi N, Zuccato C, et al. (2015) Recent trends in the gene therapy of $\beta$-thalassemia. J Blood Med 6: 69-85. 